\title{
BMJ Open Raising the topic of weight in general practice: perspectives of GPs and primary care nurses
}

\author{
Maxine Blackburn, ${ }^{1}$ Afroditi Stathi, ${ }^{1}$ Edmund Keogh, ${ }^{2}$ Christopher Eccleston ${ }^{1}$
}

To cite: Blackburn M, Stathi A, Keogh E, et al. Raising the topic of weight in general practice: perspectives of GPs and primary care nurses. BMJ Open 2015;5: e008546. doi:10.1136/ bmjopen-2015-008546

- Prepublication history and additional material is available. To view please visit the journal (http://dx.doi.org/ 10.1136/bmjopen-2015008546)

Received 20 April 2015 Revised 3 July 2015 Accepted 16 July 2015

\section{ABSTRACT}

Objective: To explore general practitioners' (GPs) and primary care nurses' perceived barriers to raising the topic of weight in general practice.

Design: A qualitative study using the Theoretical Domains Framework (TDF). 34 semistructured interviews were conducted to explore views, opinions and experiences of initiating a discussion about weight. Content and thematic analyses were used to analyse the interview transcripts.

Setting: General practices located in one primary care trust in the South West of England.

Participants: 17 GPs and 17 nurses aged between 32 and 66 years. The modal age range for GPs was 30-39 years and for nurses, $40-49$ years.

Results: Barriers were synthesised into three main themes: (1) limited understanding about obesity care, (2) concern about negative consequences, and (3) having time and resources to raise a sensitive topic. Most barriers were related to raising the topic in more routine settings, rather than when dealing with an associated medical condition. GPs were particularly worried about damaging their relationship with patients and emphasised the need to follow their patient's agenda.

Conclusions: Uncertainty about obesity, concerns about alienating patients and feeling unable to raise the topic within the constraints of a 10 min consultation, is adding to the reluctance of GPs and nurses to broach the topic of weight. Addressing these concerns through training or by providing evidence of effective interventions that are feasible to deliver within consultations may lead to greater practitioner engagement and willingness to raise the topic.

\section{INTRODUCTION}

Primary care clinicians including general practitioners (GPs) and primary care nurses have been assigned a key role in the prevention and treatment of excess weight and obesity. Recent reports produced by the Academy of Medical Royal Colleges ${ }^{1}$ and the Royal College of Physicians ${ }^{2}$ emphasise the influence that clinicians can have on their patients' health behaviours. ${ }^{1}{ }^{2}$ Evidencebased guidelines recommend that

\section{Strengths and limitations of this study}

- This article explores general practitioner (GP) and nurse barriers to raising the topic of weight in general practice, and finds that some clinicians encounter significant barriers. The identification of barriers was facilitated by drawing on a validated theoretical framework based on behaviour change theory.

- The inclusion of both GP and nurse barriers provides novel insight into the research problem.

- A strength of the study is the underpinning theoretical framework, which facilitated a broad and comprehensive approach to the identification of barriers to raising the issue. However, this could also be considered a limitation, since it precluded in-depth analysis into the nature of individual barriers and may have prevented the identification of barriers that deviated from the framework.

- A limitation of the study design is the focus on clinician beliefs and attitudes, rather than the social and moral context of the consultation, or the ways in which clinical encounters are mediated by broader social and cultural discourses surrounding obesity.

- A further limitation of the study design is the absence of theoretical concepts such as stigma and power in the analysis of our findings. While we consider these important and relevant concepts to include in research concerning obesity, it was beyond the scope of this study to include this level of analysis.

practitioners identify and classify overweight and obesity by using 'clinical judgement to decide when to measure a person's height and weight', ${ }^{3}$ and offer clinical management. ${ }^{3}{ }^{4}$ Despite these strong calls to action, evidence from patient surveys suggest that less than half of obese patients are advised by their physician to lose weight. ${ }^{5}$ Recently, in Great Britain, a cross-sectional survey of 810 overweight or obese adults found that only $17 \%$ of overweight and $42 \%$ of obese respondents recalled ever receiving health professional advice to lose weight. ${ }^{7}$ 
In order to engage GPs and nurses in supporting patients to lose weight, it is important to explore why discussions about weight loss in primary care are infrequent. Evidence to date suggests that GPs and nurses find obesity difficult to discuss and are concerned about raising the subject within the consultation. ${ }^{8}$ This research has largely focused on barriers to obesity management, particularly the provision of advice for obesity. Studies, mainly using survey and interview methods, indicate that lack of time, limited training, low expectations of success and worry of being offensive to patients prevent health professionals from playing an active role in treating obesity. ${ }^{8-10}$

It is also useful to review the barriers that health professionals experience when addressing other public health problems, such as smoking and alcohol use. Like obesity, smoking and alcohol consumption have been framed as 'lifestyle risk factors', and have been identified as sensitive matters to address in the consultation. ${ }^{11-13}$ While studies have reported some similarities in the barriers to raising these issues, for example, all relate to individual lifestyle habits and are thus potentially 'face threatening, ${ }^{11-14}$ there are also differences in the challenges of addressing such topics. Smoking is an area of public health that has received support at both a primary care and national level, resulting in increased provision of services in general practice and greater acceptance of smoking as a health threat. ${ }^{15} 16$ In addition, beneficial effects from clinician-delivered brief interventions and referral to specialist services have been established for smoking, and harmful and hazardous alcohol consumption, ${ }^{17-20}$ yet there remains a lack of evidence for effective weight loss interventions that can be delivered at a population level in primary care. ${ }^{21}{ }^{22}$ It is therefore important to recognise that obesity presents unique challenges to the primary care team.

In summary of the literature to date, there has been little exploration of the full range of barriers that may hinder clinicians from raising the topic of weight for the first time with a patient. In addition, few studies have sought the views of GPs and nurses, who both have a shared responsibility to promote weight loss and facilitate access to weight management support. ${ }^{2}$ In light of this gap, the study sought to systematically identify and describe GPs' and primary care nurses' beliefs and attitudes regarding barriers to raising the topic of weight with overweight and obese patients presenting in general practice. Raising the topic was defined as initiating a discussion about weight loss.

\section{METHOD}

Design

This was a qualitative study using semistructured interviews drawing on constructs and definitions from the Theoretical Domains Framework (TDF). ${ }^{23} 24$ The framework was judged to be a suitable conceptual tool to guide the design and analysis of the study since it enables an exploration of the full range of potential influences on behaviour and has been validated to facilitate research into implementation problems. ${ }^{24}$

The TDF is a framework based on theories of human behaviour and behaviour change, and is in line with calls for complex interventions to improve health to be informed by theory. ${ }^{25}{ }^{26}$ The TDF was developed to identify the causes of implementation difficulties and promote understanding about how to change health professional behaviour. The framework derived from the integration of 33 theories and 128 constructs from behavioural theory, resulting in 12 theoretical domains useful for categorising barriers and enablers to specific behaviours. The TDF has been used in a number of empirical studies with healthcare professionals to explore implementation problems in clinical areas, such as low back pain, ${ }^{27}$ mental health, ${ }^{28}$ smoking cessation ${ }^{29}$ and dementia, ${ }^{30}$ supporting its validity as a theoretical framework. ${ }^{24}$ Recently, a coding manual has been developed by Heslehurst $e t a l,{ }^{31}$ adapted from Michie $e t a l^{23}$ and Cane et $a l,{ }^{24}$ which lays out 12 theoretical domains used to inform the topic guide of qualitative studies and the analysis of interview transcripts (see online supplementary additional file 1).

\section{Participant selection and recruitment}

All participants gave informed consent before taking part in the interviews.

Purposive sampling was used to recruit a heterogeneous sample of GPs and nurses working within one primary care trust in the South West of England. Study information was provided at a practice manager meeting, and emails outlining the study were sent to 58 GP surgeries and to a network of sessional GPs in the local authority. This resulted in 13 GPs and 14 nurses agreeing to be interviewed after receiving further details about the study. Snowball sampling was also used to recruit participants; four GPs and three nurses were approached, either in person or via email, and all agreed to be interviewed. Prior to taking part in the study, participants were informed that interviews would involve discussion about views of obesity, role and efficacy beliefs, and the challenges involved in raising the topic of weight in general practice. Participants were recruited until no new information and understanding from the interviews occurred. ${ }^{32} 33$ As a token of appreciation, participants were offered the opportunity to claim practice level reimbursement for their time.

\section{Data collection}

A flexible interview schedule was developed based on the TDF domains and a review of empirical research literature concerning barriers to health professional prevention and management of obesity in primary care (see online supplementary additional file 2). The topic guide for the interviews began by asking participants about the factors that triggered them to broach discussions about weight loss. The remainder of the questions 
focused on the theoretical domains, to gain insight into factors hindering discussion about weight loss. Prior to interviews, the questions were piloted with three GPs and two primary care nurses, to assess clarity and focus of the interview schedule, and refined as appropriate.

Face-to-face individual interviews were conducted by the lead researcher (MB), at a time and place to suit the participant. Interview locations included general practice offices, the University of Bath and participants' homes.

Interviews lasted between 30 and 90 min. Participants were encouraged to express the barriers most salient to them and prompted to expand on views when deemed appropriate by the researcher. Interviews were digitally audiorecorded, and then transcribed verbatim by the lead researcher and an external agency with transcription expertise.

All data collection took place over January and February 2013.

\section{Data management and analysis}

Audio recordings were transcribed verbatim in Microsoft Word and then uploaded to NVivo (V.10) for coding and data organisation. A period of familiarisation with the data set by the lead researcher was followed by a process of coding whereby a priori themes directed by the interview topic guide, unexpected emergent themes and recurring viewpoints were identified. A deductive approach to content analysis ${ }^{34}$ was used to code the data to the TDF framework, whereby data were reviewed for content and correspondence to identified categories of the TDF. ${ }^{31}$ The manifest and latent content were both examined. ${ }^{35} 36$ The TDF coding framework developed by Heslehurst $e t a l^{31}$ was used to ensure code names were matched to the appropriate domains. The accuracy of this initial coding, derived from a subset of the data, was checked by other members of the research team, and then used to guide the indexing of the remaining transcripts. Following the mapping of codes to the domains of the TDF, the lower order themes were charted and organised into three salient higher order themes that manifest within the whole data set. This process was facilitated by drawing on principles of thematic analysis ${ }^{37}$ and additional behaviour change theory designed to guide the grouping of domains in the TDF into broader components. ${ }^{38}$ At the final stage of data analysis, the derived themes for GPs and nurses were compared, and similarities and differences were identified. Analysis was a recursive process that developed over time, with the lead researcher continually revisiting the data set and theoretical literature before arriving at the final themes.

\section{RESULTS}

\section{Characteristics of GPs and nurses}

Of the 17 GPs interviewed, 5 were partners, 6 were salaried ( 1 of whom was a GP assistant) and 6 were locums. Of the 17 nurses interviewed, 3 were nurse practitioners.
Nursing roles varied widely: six nurses specialised in diabetes care ( 3 of whom also carried out general practice nurse duties), three nurses specialised in chronic obstructive pulmonary disease and asthma (2 of whom also carried out general practice nurse duties), four nurses worked in emergency and minor illness roles (1 of whom also carried out general practice nurse duties), and four nurses were identified as having a generalist practice nurse role. Respondents came from rural, semirural and urban practices. Additional demographic data are presented in table 1.

Content analysis ${ }^{34}$ resulted in 25 individual barriers to raising the issue, which were then mapped to 10 behavioural domains of the TDF (table 2). Barriers identified do not apply to all clinicians but were identified as barriers for at least two clinicians. Barriers were synthesised into three higher order themes (figure 1) that manifest within the entire data set. Each theme is described and illustrated by selective quotations from respondents to aid understanding.

\section{Main themes}

Barriers were synthesised into three overriding themes during the second stage of the analysis: limited understanding about obesity care, concern about negative consequences, and lacking time and resources to deal with a sensitive issue. Each theme is described and quotations from interviewees provided to illustrate the barriers within the themes. If applicable, differences between GP and nurse barriers are highlighted in the description of each theme.

\section{Limited understanding about obesity care}

The first theme relates to two domains of the TDF: knowledge and skills. Within this theme, there was low

\begin{tabular}{|c|c|c|}
\hline & GPs & Nurses \\
\hline \multicolumn{3}{|l|}{ Sex } \\
\hline Male & 6 & \\
\hline Female & 11 & 17 \\
\hline \multicolumn{3}{|l|}{ Age (in years) } \\
\hline 30-39 & 7 & 1 \\
\hline $40-49$ & 3 & 7 \\
\hline $50-59$ & 6 & 5 \\
\hline $60-69$ & 1 & 4 \\
\hline \multicolumn{3}{|c|}{ Experience as GP/nurse in general practice (in years) } \\
\hline $0-9$ & 7 & 7 \\
\hline $10-19$ & 3 & 6 \\
\hline 20-29 & 7 & 4 \\
\hline \multicolumn{3}{|l|}{ Weight status } \\
\hline Normal (BMI 18.5-24.9 kg/m²) & 9 & 9 \\
\hline Overweight (BMI 25-29.9 kg/m²) & 7 & 4 \\
\hline Obese (BMI $30 \mathrm{~kg} / \mathrm{m}^{2}$ and above) & & 4 \\
\hline Not specified & 1 & \\
\hline
\end{tabular}


Table 2 Barriers coded to the TDF framework

\begin{tabular}{|c|c|c|c|}
\hline Behavioural domain & Barrier & GP & Nurse \\
\hline \multirow[t]{3}{*}{ Knowledge } & Lacking content knowledge of guidelines & $\sqrt{ }$ & $\sqrt{ }$ \\
\hline & Not recognising obesity as a complex medical problem & $\sqrt{ }$ & $\sqrt{ }$ \\
\hline & Uncertainty about raising the topic routinely & $\sqrt{ }$ & $\sqrt{ }$ \\
\hline \multirow[t]{2}{*}{ Skills } & Uncertainty about how to raise the topic sensitively & $\sqrt{ }$ & $\sqrt{ }$ \\
\hline & $\begin{array}{l}\text { Uncertainty about how to raise the topic when patient is not consulting with } \\
\text { related problem }\end{array}$ & $\sqrt{ }$ & $\sqrt{ }$ \\
\hline \multirow[t]{4}{*}{ Beliefs about consequences } & Potential to damage the doctor-patient relationship & $\sqrt{ }$ & \\
\hline & Concern that patient will feel alienated and disengage from healthcare & $\sqrt{ }$ & $\sqrt{ }$ \\
\hline & Beliefs about negative responses & $\sqrt{ }$ & $\sqrt{ }$ \\
\hline & Potential to 'open a can of worms' & $\sqrt{ }$ & $\sqrt{ }$ \\
\hline Beliefs about capabilities & Feeling ineffective at helping patients with weight loss & $\sqrt{ }$ & $\sqrt{ }$ \\
\hline Motivation & Desire to maintain a positive, non-judgemental relationship with patient & $\sqrt{ }$ & \\
\hline Competing goals & $\begin{array}{l}\text { Prioritising other areas of patient care } \\
\text { Prioritising other public health concerns }\end{array}$ & $\sqrt{ }$ & $\sqrt{ }$ \\
\hline \multirow[t]{4}{*}{ Emotion } & Fear of upsetting patients & $\sqrt{ }$ & $\sqrt{ }$ \\
\hline & Feeling awkward/uncomfortable raising the issue & $\sqrt{1}$ & $\sqrt{1}$ \\
\hline & Hopelessness & $\sqrt{ }$ & $\sqrt{ }$ \\
\hline & Frustration & $\sqrt{ }$ & $\sqrt{ }$ \\
\hline \multirow{3}{*}{$\begin{array}{l}\text { Professional role and } \\
\text { identification }\end{array}$} & Threat to professional reputation & $\sqrt{ }$ & \\
\hline & Impact of own weight status & $\sqrt{ }$ & $\sqrt{ }$ \\
\hline & Personal feelings about advocating weight loss & $\sqrt{ }$ & $\sqrt{ }$ \\
\hline \multirow{3}{*}{$\begin{array}{l}\text { GP practice and available } \\
\text { resources }\end{array}$} & Having time to open up a sensitive issue & $\sqrt{ }$ & $\sqrt{ }$ \\
\hline & Feeling as if there's nothing to offer patients & $\sqrt{ }$ & $\sqrt{ }$ \\
\hline & No continuity of care with patients & $\sqrt{ }$ & $\sqrt{ }$ \\
\hline \multirow[t]{2}{*}{ Social influences } & Adhering to the patient's agenda & $\sqrt{ }$ & \\
\hline & Perceptions about patient receptiveness to advice & $\sqrt{ }$ & $\sqrt{ }$ \\
\hline
\end{tabular}

awareness of the contents of any guidelines around raising the topic. Clinicians expressed beliefs that there is no standardised approach to raising the issue, and acknowledged that they relied on a range of sources to provide weight loss advice, including personal experience and media sources. Divergent opinions around when to raise the topic were apparent with some clinicians believing it inappropriate to raise the topic in routine consultations. While some practitioners described obesity as a complex medical condition requiring medical support, a minority of interviewees explicitly questioned whether obesity was a medical problem. These clinicians expressed the opinion that although obesity had medical implications, it was largely a social problem that may be better tackled outside primary care. Other clinicians described concern about creating obesity into a medical problem.

"Is obesity a medical problem? It has medical implications, I don't think necessarily it's a medical problem, and I think sometimes it's better being dealt with outside the NHS, you know, because it has a lot of, there are a lot of other factors that cause people to be heavier than perhaps they need to come to a medical practice for." (Participant 25, Nurse)

Uncertainty about how to initiate weight loss discussions when patients were presenting with problems unrelated to excess weight were discussed. In these consultations, the identification of excess weight was considered to be particularly problematic, and there was uncertainty about the appropriate language and terminology to use. Negative societal views about obesity contributed to the difficulty of framing a discussion about weight loss in a positive and constructive light.

"Just bringing it up....how do you bring it up, when they've come in about a cold? It's really difficult isn't it because you know we've all got to be very PC [ politically correct] and people get very hurt even with medical terms like obesity or overweight, it can be really challenging." (Participant 13, GP)

Feeling unable to help patients with weight loss was identified as a barrier for a proportion of clinicians. In contrast, other respondents emphasised the value of being able to offer advice and support. Nurses who had been involved in training and research studies discussed their increased confidence and perceived effectiveness of supporting patients with weight loss, suggesting a lack of knowledge and skills around obesity management may have acted as a barrier to raising the topic previously.

"I don't find it that difficult any more. I think I may be used to but I think again because we took part in the research study and things, I think I find it a bit easier. I 


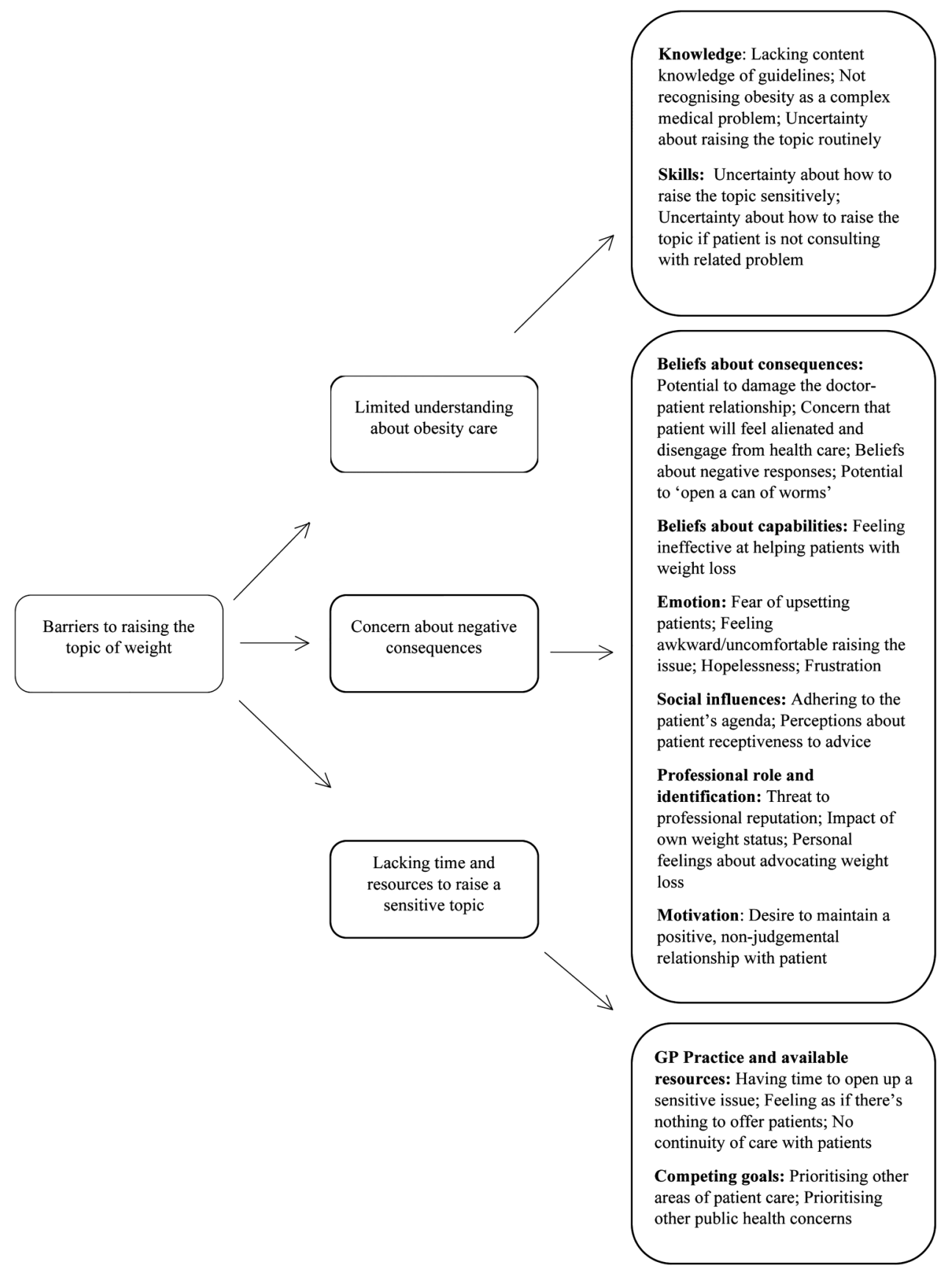

Figure 1 Barriers to raising the topic synthesised into three analytic themes (GP, general practitioner).

think I find it easier in the fact that before-it's okay to raise a patient's issue of weight, but if you've not got any advice to give them then what's the point? So yeah, so I don't mind so much now because at least I can sort of steer them, give them a bit of advice." (Participant 28, Nurse)

\section{Concern about negative consequences}

Negative consequences of raising the topic of weight relate to six domains of the TDF: beliefs about consequences; beliefs about capabilities; emotion; social influences; professional role and identification; and motivation. The potential for patients to feel blamed, persecuted and further stigmatised about their weight was widely discussed. Concerns were expressed that a narrow focus on weight at the expense of other health problems could be counterproductive and alienate patients from consulting in the future.

"The last thing you want to do is completely disenfranchise a patient such that they're very reluctant to see anybody, that'd be counterproductive. We are very time limited but I don't think it takes long to raise the issue of weight so I think the main thing would be losing the patient trust and the patient's engagement." (Participant $1, \mathrm{GP})$

Several of the barriers within this theme were unique to GPs, who expressed concern that raising the topic of 
weight conflicted with their desire to maintain a nonjudgemental relationship with patients. Raising the topic of weight was viewed as a threat to professional reputation, with acknowledgement by some GPs that they did not want to become known as the 'nagging doctor'. GPs also expressed concern about deviating from the patients' agenda, stressing the importance of treating a patients' presenting problem and meeting expectations.

"I think patients having confidence that they can come and talk to their GP about anything and they won't be judged ... if I have to not talk about something or talk about something very sort of gently in order to preserve my reputation as being non-judgmental then I will do that." (Participant 15, GP)

In regard to a health professional's own weight status and personal health beliefs, no clear pattern emerged in relation to whether these factors acted as barriers to raising the topic. Having a body mass index (BMI) in the normal weight range was viewed as a barrier by some clinicians due to beliefs that patients would perceive them to lack empathy. Having a BMI in the obese range was viewed as a barrier by several nurses who acknowledged feeling uncomfortable raising the issue due to the personal nature of such discussions, the difficulties of weight loss and uncertainty about the credibility of their message.

"Being a rather larger person myself, I find it sometimes a little bit sensitive to say, 'You really ought to lose some weight', when, actually, the same person could be saying it back to me." (Participant 23, Nurse).

\section{Lacking time and resources to deal with a sensitive issue}

The lack of time available in a consultation was judged to hinder the opportunity for clinicians to engage in sensitive discussion about weight loss. This theme consisted of two domains of the TDF: GP practice and available resources, and competing goals within the consultation. Owing to the limited time and the need to run to schedule for other patients waiting in the surgery, clinicians perceived they lacked time to initiate a discussion about weight loss. This barrier was particularly salient for clinicians working within 10 min consultations and when patients were attending for medical problems unrelated to excess weight. Nurses with longer appointment times, and clinicians working in practices and/or roles that facilitated continuity of care with patients, emphasised their confidence in having adequate time to begin a dialogue about weight loss and emphasised that discussions could continue over a series of visits.

"I think often it is time because you have a patient that comes, you have ten minute appointments, there is not really much scope, patients are not happy to wait generally in general practice so even if you think you should really mention this, you know it's going to be time consuming, it's not a quick consultation about weight." (Participant 14, GP)
Views about services to offer overweight patients differed between clinicians. Some clinicians expressed optimism about signposting to groups such as Slimming World or Exercise on Referral, comparing this with being able to offer a prescription. Others felt these schemes lacked evidence of long-term success and expressed ambivalence. Clinicians perceiving themselves unable to offer any assistance or constructive support to patients expressed hopelessness and frustration.

"It's just not something I enjoy doing because people do get very offended and feel very judged and also I feel I haven't got, having brought it up, I haven't got huge amounts of resources then to offer people to help them with it." (Participant 12, GP)

Competing goals within the consultation were perceived to hinder the opportunity to raise the issue. Clinicians asserted that their main concern was to deal with the patients presenting problem, which often restricted the opportunity to raise weight as an additional issue. Factors relating to individual patient needs and preferences, such as the patient's presenting illness and the broader social context of a patient's life, were also judged to inhibit the opportunity for clinicians to initiate a discussion about weight loss. Raising other public health issues, such as smoking, was identified as a barrier mainly discussed by nurses.

"That's the trouble isn't it, it's the conflict of time for all the other things that we're supposed to do in a ten minute consultation, of which probably smoking cessation comes quite high on the sort of health promotion thing...and alcohol, of course, that's another." (Participant 34, Nurse).

\section{DISCUSSION}

The purpose of this study was to systematically map the barriers to raising the topic of weight in general practice by capturing the perspectives of GPs and primary care nurses. Using behaviour change theory, barriers were mapped into 10 domains on the TDF and synthesised into three higher order analytic themes. Future interventions wishing to change the behaviour of GPs and nurses may wish to target the identified domains of the TDF that are proposed to be mediators of behaviour change. $^{23} 24$

This study confirms that the majority of barriers relate to raising the topic of weight when patients are consulting with a medical problem that is not considered to be linked to obesity. ${ }^{8} 39$ The majority of clinicians working in generalist roles do not discuss weight with patients as a routine part of clinical practice due to beliefs that it is inappropriate, unfeasible or unacceptable to patients. While clinicians said they were more likely to discuss weight with patients in the context of a weight-related health problem, the multitude of barriers in any single consultation, including factors that appear to be 
distinctive to obesity, such as stigma, may prevent clinicians from discussing weight despite recognising the need to. An important finding is the uncertain knowledge demonstrated by GPs and nurses about obesity as a medical condition that should be prevented and treated in primary care. This suggests that, despite increased attention towards the role of primary care in treating obesity, there are still gaps in the implementation of this knowledge. A proportion of clinicians remain ambivalent about their role in helping patients with weight loss. It has been reported elsewhere that medical professionals view lifestyle change as a personal choice, ${ }^{40}$ and believe obesity may be better addressed by politicians at a societal level. ${ }^{9}{ }^{41}{ }^{42}$ Although the findings of the current study suggest that the health and economic consequences of obesity are recognised, knowledge surrounding how obesity should be treated remains disputed and inconsistent among practitioners. These views may also reflect the limited evidence-base for effective primary care-led weight loss interventions, ${ }^{43}$ as well as the controversy and uncertainty surrounding obesity on a national level. ${ }^{44} 45$

A novel insight to emerge is the personal dimension of discussing weight loss, which appears to influence clinician views. The majority of GPs and nurses expressed the view that their own body weight and personal beliefs about weight loss could act as barriers to raising the issue. Research has highlighted that clinicians with a BMI classified in the overweight or obese weight range experience more barriers to offering weight loss advice than clinicians with a BMI categorised in the normal weight range. ${ }^{46}{ }^{47}$ However, this study suggested a more complex and nuanced picture between clinician weight status and attitudes, with normal weight and overweight clinicians expressing the view that their own weight status was a potential barrier to raising the issue. At a time when negative views and attitudes towards people with excess weight are evident in society, ${ }^{48}$ it is important to explore how clinicians perceive the increased pressure to deliver weight loss advice, and understand the influence their personal values and experiences of weight loss have on this task.

\section{Key differences between GPs and nurses}

In comparison with nurses, GPs expressed greater concern about the potential to damage their relationship with a patient and divert the consultation away from the patient's agenda. GPs were particularly cautious about raising the topic in routine consultations due to the risk of losing patient trust and damaging their own professional reputation. Other research has emphasised the centrality GPs place on abiding by their patient's agenda ${ }^{40} 50$ as well as highlighting that doctors may fail to take account of unvoiced agendas ${ }^{51}$ or recognise elements of the patient's agenda, particularly those of a social and emotional nature. ${ }^{52} 53$

\section{Strengths and limitations of the study}

A key strength of the research is the underpinning theoretical framework guiding the design and analysis of the study. Using constructs drawn from theories of behaviour change facilitated the identification of the factors influencing health professional behaviour. The qualitative design of the study revealed the nuances and tensions inherent in managing obesity in primary care. The recruitment of a diverse sample of GPs and primary care nurses with a wide range of experience and specialities is a further strength of this study. The inclusion of locum GPs and the diversity of nursing roles ensured the identification of a wide variety of barriers.

Limitations include the nature of recruitment, with the majority of GPs and nurses actively volunteering to this study. This may have resulted in recruiting clinicians with more interest in identifying and discussing the issue of raising the topic of weight and obesity than is typical. The sample of health professionals is also drawn from one location. Although we ensured that the interviewees operate in diverse socioeconomic environments, recruitment from other sites might have revealed new dimensions. In this study, we explored barriers to raising the topic of weight in consultations focusing on related as well as unrelated problems. Since findings of our study highlight the particular difficulties of broaching the topic in consultations about unrelated problems, future research could focus mainly on exploring this in more detail. The study did not recruit any GPs who self-reported a BMI in the obese range, which may have excluded gaining more insight into barriers related to a GPs own weight status. For this reason, the study does not claim that having a BMI in the obese range is a barrier for nurses only, as it is possible that it is also a barrier for GPs. Since GPs and nurses with a BMI in the normal range both considered their weight status a potential barrier to raising the topic, the study identified 'impact of own weight status' as a barrier on a broader level. Finally, variation in interview length occurred due to constraints and demands on clinician time.

\section{Strengths and limitations of this study in relation to other studies}

While a strength of this study is the comprehensive coverage it has given to a multitude of barriers, it precluded the in-depth investigation into each barrier or behavioural domain. Other studies investigating interactions concerning obesity have incorporated theoretical concepts such as stigma ${ }^{54} 55$ and shame,${ }^{14}$ while taking a critical stance to the use of language. These methods give rich and contextualised findings, provide insight into meanings and power dynamics, and go beyond the individual clinician to incorporate broader sociopolitical influences. ${ }^{56}$ Since the purpose of the TDF is to identify behavioural domains that warrant further investigation, ${ }^{24}$ findings of this study can facilitate 
future research as they can be used to indicate the selection of relevant theory to generate more detailed understanding.

A further limitation of this study in comparison to other qualitative research conducted in general practice, is the deductive nature of enquiry taken and the implications this has for how the research was conducted, and the resultant findings. The TDF is based on behaviour change theories that carry assumptions about the relationship between cognitions and behaviour, and that focus on individual-level beliefs and attitudes in relation to a discrete behaviour. It can be argued that this method is inadequate to capture the dynamic and interactional aspects of practice. ${ }^{58}$ Other research exploring how topics such as smoking and alcohol are introduced into the consultation have emphasised the process of negotiation inherent in these consultations, and highlighted the importance of the context in which advice is given, including the interactional and practical constraints on practice. ${ }^{60}{ }^{61}$ Although the targeting of specific barriers by eliciting clinician beliefs can be considered a strength of the current study, a method better able to incorporate the socially situated and interactive nature of barriers in the context of general practice is needed in future enquiry.

Future research may wish to explore insights from the study that the framework failed to adequately capture. For example, it was noted that clinicians held conflicting views, particularly regarding the framing of obesity as a medical condition, suggesting ambivalence and discomfort around this area of care. Furthermore, it was possible to detect implicit frustration regarding the perceived lack of responsibility and denial/defensiveness demonstrated by patients. Another interesting insight was the uncertainty around initiating discussions about weight with patients presenting with emotional and/or mental health problems, including low self-esteem, depression and body image concerns, with many clinicians expressing reluctance to discuss weight in such situations. Given that obesity is associated with an increased risk of depression and reduced psychological well-being, ${ }^{62}$ a potential mechanism of this association being weight stigma, ${ }^{63}{ }^{64}$ there may be a significant number of patients who are not offered support to lose weight, or with whom weight-related concerns are not discussed, suggesting compromised care for these patients.

\section{Implications}

The findings of this study provide a detailed insight into how practitioners can be supported to discuss weight loss with patients. Most apparent is the need to address the uncertain knowledge about obesity as a complex medical condition and to clarify the role of primary care professionals in the management, and potentially prevention, of obesity. Concern about negative consequences of raising the topic suggests that clinicians need support to engage with patients about weight in a non- stigmatising and non-harmful way. Offering training and education that includes the views of individuals with obesity and provides health professionals with a comprehensive understanding of the stigma and psychological impacts of obesity, is just one way that health professionals could be empowered in this area of practice. In addition, evidence of brief interventions that are feasible to be implemented in primary care settings and that target multilevel barriers is required. Finally, lessons from other areas of public health could be drawn on, particularly smoking, where clinicians are equipped with smoking cessation services and pharmacological treatment, and are incentivised by the Quality and Outcomes Framework as part of a comprehensive strategy to lower rates of smoking in the population. ${ }^{65}{ }^{66}$ Although such changes may encourage practitioners to raise the topic routinely, this study confirms that there are challenges unique to discussing obesity, particularly weight stigma, that need to be further explored and targeted in future research.

\section{CONCLUSION}

Raising the topic of weight within a general practice consultation is a complex endeavour for GPs and practice nurses to negotiate with their patients. Uncertainty about how and when to raise the topic of weight, and the threat of alienating and/or upsetting patients, are contributing to an unease and a lack of motivation by healthcare professionals to identify weight as an issue. Furthermore, competing demands and limited time available in brief consultations limit the opportunity for intervention.

Contributors MB, AS, EK and CE designed the study. MB wrote the first draft of the paper and is the guarantor. MB undertook all of the interviews, and transcribing and coding of the transcripts. AS checked the initial coding of a selection of the transcripts conducted by MB. Analysis was discussed at regular team meetings by all the authors; and all of them contributed to writing of the manuscript.

Funding The study is part of a $\mathrm{PhD}$ thesis undertaken by $\mathrm{MB}$ at the University of Bath, funded by Wiltshire Public Health.

Competing interests $\mathrm{MB}$ is undertaking a postgraduate degree funded by Wiltshire Public health. MB was a member of the former Royal College of General Practitioners (RCGP) Nutrition Group and contributed to the British Psychological Society shared response to a consultation on the update of NICE clinical guideline 43: Identification, assessment and management of overweight and obesity in children, young people and adults.

Ethics approval The study complied with the code of practice on ethical standards set by the Research Ethics Approval Committee for Health at the University of Bath: EP 12/13 1. Permission was granted by the local National Health Services Research and Development unit (R\&D approval number 2012/ 065).

Provenance and peer review Not commissioned; externally peer reviewed.

Data sharing statement No additional data are available.

Open Access This is an Open Access article distributed in accordance with the Creative Commons Attribution Non Commercial (CC BY-NC 4.0) license, which permits others to distribute, remix, adapt, build upon this work noncommercially, and license their derivative works on different terms, provided the original work is properly cited and the use is non-commercial. See: http:// creativecommons.org/licenses/by-nc/4.0/ 


\section{REFERENCES}

1. Academy of Medical Royal Colleges. Measuring up. The medical profession's prescription for the nation's obesity crisis. Academy of Medical Royal Colleges, 2013. http://www.aomrc.org.uk/projects/ obesity-steering-group.html (accessed 15 Jan 2014).

2. Royal College of Physicians. Action on obesity: comprehensive care for all Royal College of Physicians. Report of a working party. Royal College of Physicians, 2013. https://www.rcplondon.ac.uk/ resources/action-obesity-comprehensive-care-all (accessed Apr 2014).

3. National Institute for Health and Care Excellence (NICE). Obesity: guidance on the prevention, identification, assessment and management of overweight and obesity in adults and children. NICE, 2014. http://www.nice.org.uk/ (accessed 1 Sep 2014).

4. National Institute for Health and Care Excellence (NICE). Managing overweight and obesity in adults-lifestyle weight management services. NICE, 2014. http://www.nice.org.uk/guidance/ph53 (accessed 18 Aug 2014).

5. Abid O, Galuska D, Khan LK, et al. Are healthcare professionals advising obese patients to lose weight? A trend analysis. Med Gen Med 2005;7:10.

6. Kirk S, Tytus R, Tsuyuki R, et al. Weight management experiences of overweight and obese Canadian adults: findings from a national survey. Chronic Dis Inj Can 2012;32:63-9.

7. Jackson SE, Wardle J, Johnson F, et al. The impact of a health professional recommendation on weight loss attempts in overweight and obese British adults: a cross-sectional analysis. BMJ Open 2013;3:e003693.

8. Michie S. Talking to primary care patients about weight: a study of GPs and practice nurses in the UK. Psychol Health Med 2007;12:521-5.

9. Epstein L, Ogden J. A qualitative study of GPs' views of treating obesity. Br J Gen Pract 2005;55:750-4.

10. Brown I, Stride C, Psarou A, et al. Management of obesity in primary care: nurses' practices, beliefs and attitudes. J Adv Nurs 2007;59:329-41.

11. Larsson US, Säljö R, Aronsson K. Patient-doctor communication on smoking and drinking: lifestyle in medical consultations. Soc Sci Med 1987;25:1129-37.

12. Beich A, Gannik D, Malterud K. Screening and brief intervention for excessive alcohol use: qualitative interview study of the experiences of general practitioners. BMJ 2002;325:870-2.

13. Bell K, Salmon A, McNaughton D. Alcohol, tobacco, obesity and the new public health. Crit Public Health 2011;21:1-8.

14. Guassora AD, Reventlow S, Malterud K. Shame, honor and responsibility in clinical dialog about lifestyle issues: a qualitative study about patients' presentations of self. Patient Educ Couns 2014;97:195-9.

15. Brownson RC, Jackson-Thompson J, Wilkerson JC, et al. Demographic and socioeconomic differences in beliefs about the health effects of smoking. Am J Public Health 1992;82: 99-103.

16. Royal College of Physicians. Fifty years since smoking and health. Progress, lessons and priorities for a smoke-free UK. Report of conference proceedings. London: RCP, 2012. https://www. rcplondon.ac.uk/sites/default/files/fifty-years-smoking-health.pdf (accessed 30 Jun 2015).

17. National Institute for Health and Care Excellence (2006). Brief interventions and referral for smoking cessation. http://www.nice.org uk/guidance/ph1 (accessed 30 Jun 2015).

18. National Institute for Health and Care Excellence (2010). Alcohol-use disorders: preventing harmful drinking. http://www.nice. org.uk/guidance/ph24 (accessed 30 Jun 2015).

19. Aveyard P, Begh R, Parsons A, et al. Brief opportunistic smoking cessation interventions: a systematic review and meta-analysis to compare advice to quit and offer of assistance. Addiction 2012;107:1066-73.

20. Kaner EF, Beyer F, Dickinson HO, et al. Effectiveness of brief alcohol interventions in primary care populations. Cochrane Database Syst Rev 2007;(2):CD004148.

21. Booth HP, Prevost AT, Gulliford MC. Access to weight reduction interventions for overweight and obese patients in UK primary care: population-based cohort study. BMJ open 2015;5: e006642.

22. Lewis A, Jolly K, Adab P, et al. A brief intervention for weight management in primary care: study protocol for a randomized controlled trial. Trials 2013;14:393.

23. Michie S, Johnston M, Abraham C, et al. Making psychological theory useful for implementing evidence based practice: a consensus approach. Qual Saf Health Care 2005;14: 26-33.
24. Cane J, O'Connor D, Michie S. Validation of the Theoretical Domains Framework for use in behaviour change and implementation research. Implement Sci 2012;7:37.

25. Campbell M, Fitzpatrick R, Haines A, et al. Framework for design and evaluation of complex interventions to improve health. BMJ 2000;321:694-6.

26. Craig $\mathrm{P}$, Dieppe $\mathrm{P}$, Macintyre $\mathrm{S}$, et al. Developing and evaluating complex interventions: the new Medical Research Council guidance. BMJ 2008;337:a1655

27. Bussières A, Patey A, Francis J, et al. Identifying factors likely to influence compliance with diagnostic imaging guideline recommendations for spine disorders among chiropractors in North America: a focus group study using the Theoretical Domains Framework. Implement Sci 2012;7:82.

28. Michie S, Pilling S, Garety P, et al. Difficulties implementing a mental health guideline: an exploratory investigation using psychological theory. Implement Sci 2007;2:1-8.

29. Beenstock J, Sniehotta FF, White M, et al. What helps and hinders midwives in engaging with pregnant women about stopping smoking? A cross-sectional survey of perceived implementation difficulties among midwives in the northeast of England. Implement Sci 2012;7:36

30. Murphy K, O'Connor DA, Browning CJ, et al. Understanding diagnosis and management of dementia and guideline implementation in general practice: a qualitative study using the Theoretical Domains Framework. Implement Sci 2014;9:31.

31. Heslehurst N, Newham J, Maniatopoulos G, et al. Implementation of pregnancy weight management and obesity guidelines: a meta-synthesis of healthcare professionals' barriers and facilitators using the Theoretical Domains Framework. Obes Rev 2014;15:462-86.

32. Francis $\mathrm{JJ}$, Johnston $\mathrm{M}$, Robertson $\mathrm{C}$, et al. What is an adequate sample size? Operationalising data saturation for theory-based interview studies. Psychol Health 2010;25:1229-45.

33. Guest G, Bunce A, Johnson L. How many interviews are enough? An experiment with data saturation and variability. Field Methods 2006;18:59-82

34. Elo $\mathrm{S}$, Kyngäs $\mathrm{H}$. The qualitative content analysis process. J Adv Nurs 2008;62:107-15.

35. Patton MQ. Qualitative evaluation and research methods. 2nd edn California. Sage, 2002.

36. Silverman D. Interpreting qualitative data. 4th edn. London. Sage Publications Ltd, 2011.

37. Braun V, Clarke V. Using thematic analysis in psychology. Qual Res Psychol 2006;3:77-101.

38. Michie S, van Stralen MM, West R. The behaviour change wheel: a new method for characterising and designing behaviour change interventions. Implement Sci 2011;6:42

39. Phillips K, Wood F, Kinnersley P. Tackling obesity: the challenge of obesity management for practice nurses in primary care. Fam Pract 2013;31:51-9.

40. Chisholm A, Hart J, Lam V, et al. Current challenges of behavior change talk for medical professionals and trainees. Patient Educ Couns 2012;87:389-94.

41. Gunther S, Guo F, Sinfield P, et al. Barriers and enablers to managing obesity in general practice: a practical approach for use in implementation activities. Qual Prim Care 2012;20:93-103.

42. Ogden J, Flanagan Z. Beliefs about the causes and solutions to obesity: a comparison of GPs and lay people. Patient Educ Couns 2008;71:72-8

43. Lewis $A$, Aveyard $P$, Jebb $S$. Brief interventions for weight loss in primary care. Curr Obes Rep 2013;2:341-7.

44. Godlee F. Nutrition matters. BMJ 2014;349:g7255

45. Lucan SC, DiNicolantonio JJ. How calorie-focused thinking about obesity and related diseases may mislead and harm public health. An alternative. Public Health Nutr 2015;18:571-81.

46. Bleich SN, Bandara S, Bennett WL, et al. Impact of non-physician health professionals' BMI on obesity care and beliefs. Obesity (Silver Spring) 2014;22:2476-80.

47. Zhu D, Norman I, While A. The relationship between health professionals' weight status and attitudes towards weight management: a systematic review. Obes Rev 2011;12:459-69.

48. Brewis AA, Wutich A, Falletta-Cowden A. Body norms and fat stigma in global perspective. Curr Anthropol 2011;52:269-76.

49. Kirk SF, Price SL, Penney TL, et al. Blame, shame, and lack of support: a multilevel study on obesity management. Qual Health Res 2014;24:790-800.

50. Chew-Graham CA, May CR, Roland MO. The harmful consequences of elevating the doctor-patient relationship to be a primary goal of the general practice consultation. Fam Pract 2004;21:229-31. 
51. Barry CA, Bradley CP, Britten N, et al. Patients' unvoiced agendas in general practice consultations: qualitative study. BMJ 2000;320:1246-50.

52. Campion P, Butler N, Cox A. Principle agendas of doctors and patients in general practice consultations. Fam Pract 1992;9:181-90.

53. Salmon P, Sharma N, Valori R, et al. Patients' intentions in primary care: relationship to physical and psychological symptoms, and their perception by general practitioners. Soc Sci Med 1994;38:585-92.

54. Grønning I, Scambler G, Tjora A. From fatness to badness: the modern morality of obesity. Health 2012;17:266-83.

55. Throsby K. "How could you let yourself get like that?" Stories of the origins of obesity in accounts of weight loss surgery. Soc Sci Med 2007:65:1561-71.

56. Burr V. Social constructionism. 2nd edn. East Sussex: Routledge, 2003.

57. Parker I. Discourse dynamics: critical analysis for social and individual psychology. London: Routledge, 1992.

58. Greenhalgh T, Swinglehurst D. Studying technology use as social practice: the untapped potential of ethnography. BMC Med 2011;9:45
59. Reckwitz A. Toward a theory of social practices a development in culturalist theorizing. Eur J Soc Theory 2002;5:243-63.

60. Rapley T, May C, Kaner EF. Still a difficult business? Negotiating alcohol-related problems in general practice consultations. Soc Sci Med 2006:63:2418-28.

61. Pilnick A, Coleman T. "I'll give up smoking when you get me better": patients' resistance to attempts to problematise smoking in general practice (GP) consultations. Soc Sci Med 2003;57:135-45.

62. Luppino FS, de Wit LM, Bouvy PF, et al. Overweight, obesity, and depression: a systematic review and meta-analysis of longitudinal studies. Arch Gen Psychiatry 2010;67:220-9.

63. Schwartz MB, Brownell KD. Obesity and body image. Body Image 2004;1:43-56.

64. Stunkard AJ, Faith MS, Allison KC. Depression and obesity. Biol Psychiatry 2003;54:330-7.

65. Aveyard P. Benefits of medical treatment of obesity. Lancet 2011;377:903.

66. Stead LF, Bergson G, Lancaster T. Physician advice for smoking cessation. Cochrane Database Syst Rev 2008;(2):CD000165. 